\title{
Dwarfism with joint laxity in Friesian horses is associated with a splice site mutation in B4GALT7
}

Peter A. Leegwater ${ }^{1 *}$ (D, Manon Vos-Loohuis ${ }^{1}$, Bart J. Ducro², Iris J. Boegheim ${ }^{1,3}$, Frank G. van Steenbeek', Isaac J. Nijman ${ }^{4}$, Glen R. Monroe ${ }^{4}$, John W. M. Bastiaansen², Bert W. Dibbits², Leanne H. van de Goor ${ }^{5}$, Ids Hellinga ${ }^{6}$, Willem Back ${ }^{3,7}$ and Anouk Schurink ${ }^{2}$

\begin{abstract}
Background: Inbreeding and population bottlenecks in the ancestry of Friesian horses has led to health issues such as dwarfism. The limbs of dwarfs are short and the ribs are protruding inwards at the costochondral junction, while the head and back appear normal. A striking feature of the condition is the flexor tendon laxity that leads to hyperextension of the fetlock joints. The growth plates of dwarfs display disorganized and thickened chondrocyte columns. The aim of this study was to identify the gene defect that causes the recessively inherited trait in Friesian horses to understand the disease process at the molecular level.

Results: We have localized the genetic cause of the dwarfism phenotype by a genome wide approach to a $3 \mathrm{Mb}$ region on the p-arm of equine chromosome 14. The DNA of two dwarfs and one control Friesian horse was sequenced completely and we identified the missense mutation ECA14:9.4535550C > T that cosegregated with the phenotype in all Friesians analyzed. The mutation leads to the amino acid substitution p.(Arg17Lys) of xylosylprotein beta 1,4-galactosyltransferase 7 encoded by B4GALT7. The protein is one of the enzymes that synthesize the tetrasaccharide linker between protein and glycosaminoglycan moieties of proteoglycans of the extracellular matrix. The mutation not only affects a conserved arginine codon but also the last nucleotide of the first exon of the gene and we show that it impedes splicing of the primary transcript in cultured fibroblasts from a heterozygous horse. As a result, the level of B4GALT7 mRNA in fibroblasts from a dwarf is only $2 \%$ compared to normal levels. Mutations in B4GALT7 in humans are associated with Ehlers-Danlos syndrome progeroid type 1 and Larsen of Reunion Island syndrome. Growth retardation and ligamentous laxity are common manifestations of these syndromes.

Conclusions: We suggest that the identified mutation of equine B4GALT7 leads to the typical dwarfism phenotype in Friesian horses due to deficient splicing of transcripts of the gene. The mutated gene implicates the extracellular matrix in the regular organization of chrondrocyte columns of the growth plate. Conservation of individual amino acids may not be necessary at the protein level but instead may reflect underlying conservation of nucleotide sequence that are required for efficient splicing.
\end{abstract}

Keywords: Proteoglycan, Growth retardation, Hypermobile joints, Galactosyltransferase I, Linkeropathy, Genome, Equus caballus, Extracellular matrix

\footnotetext{
* Correspondence: p.a.jleegwater@uu.nl

${ }^{1}$ Department of Clinical Sciences of Companion Animals, Faculty of

Veterinary Medicine, Utrecht University, PO Box 80154NL-3508 TD Utrecht,

The Netherlands

Full list of author information is available at the end of the article
} 


\section{Background}

A dwarfism trait has been segregating in the Friesian horse breed for decades [1], (OMIA 000299-9796 [2]). Characteristic for the trait is the physeal growth retardation of limbs and ribs, resulting in a disproportionate form of dwarfism. The affected horses exhibit hyperextension of the fetlock joints of all limbs with varying severity. Flexor tendon laxity, which is often seen in newborn foals of all breeds, fails to recover in dwarf foals and instead tends to increase further during aging. As a consequence, these dwarf Friesians develop an abnormal gait in which the limbs undergo extreme outward rotation at the level of carpus and hocks. The ribcage is abnormal in most cases with thickened and $\mathrm{S}$-shaped costochondral junctions, leading to an inward protrusion of the chest at the level of Th10-16 (Fig. 1b,c). Mature dwarfs have a head of the same size as unaffected horses, a broader chest with narrowing at the costochondral junction, a disproportionally long back and abnormally short limbs. The abdomen has a weak and rounded appearance, and the musculature over the body is poorly developed. Involvement of the hypothalamicpituitary growth axis in the pathogenesis of the condition has been excluded [3].

A monogenic recessive mode of inheritance is most likely and, considering the breed structure with a small number of founders and narrow population bottlenecks, it is expected that the dwarfs are homozygous for the responsible gene mutation [4]. A genome wide association study of 10 cases and 10 control Friesian horses has been reported earlier [4]. The dwarfism locus was assigned to the telomeric region of the p-arm of chromosome 14 (ECA14), although genome wide significance was not reached. The aim of the present study was to confirm and further define the critical chromosome region of the locus and to identify the responsible

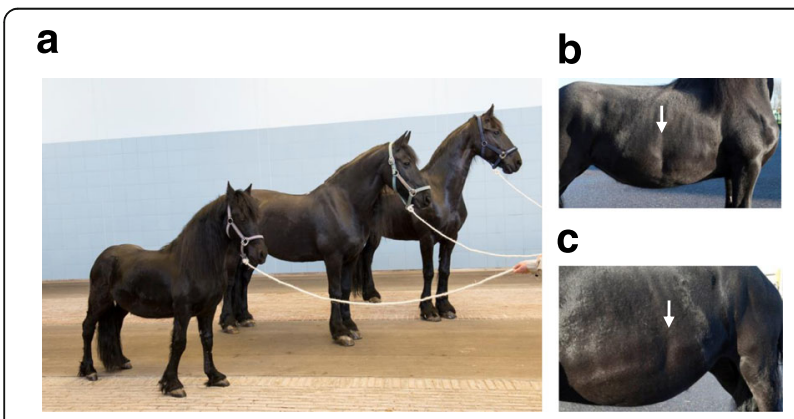

Fig. 1 Dwarfism in the Friesian horse breed. a A female dwarf next to two normal female Friesian horses. The dwarf has a height at the withers of $1.12 \mathrm{~m}$; the horse in the middle has a height of $1.54 \mathrm{~m}$, which is close to the minimum allowed by the breed standard (1.53 m); the horse on the right has a height of $1.66 \mathrm{~m}$. $\mathbf{b}$ and $\mathbf{c}$ Photographic illustrations of the typical pectus excavatum phenotype in the Friesian dwarf of the right $\mathbf{b}$ and the left side $\mathbf{c}$ gene mutation. The identification of the gene enables the comparison of the phenotype across species and enhances the understanding of the processes of growth and development.

\section{Results}

\section{Gene mapping}

To substantiate the localization of the dwarfism gene on chromosome ECA14 we performed a genome wide comparison of a group of dwarfs with a group of controls from the Friesian horse breed. The allelic association reached genome wide significance in the telomeric region of the p-arm of ECA14 with a Bonferroni corrected Pgenome $=2.90 \times 10^{-19}$ for BIEC2-239391 at position 3776009 , the SNP that was most significantly associated with dwarfism (Fig. 2a). In total 35 SNPs passed the Bonferroni corrected significance level $\left(1.68 \times 10^{-6}\right)$ and all were located on ECA14 between positions 1 and 9510581. Inspection of the genotypes of the individual horses in the region showed that only the dwarfs shared a haplotype of $3 \mathrm{Mb}$ homozygously, confirming that the phenotype originated from a single founder (Fig. 2b). The genotypes clearly pointed to recombination events that were evident in several cases and that placed the critical region between positions 3151847 and 6229282 on ECA14. According to the annotation release 101 of the NCBI of the equine reference genome the critical region contained 66 genes [5].

\section{DNA sequence analysis}

Full genome DNA sequence data was generated of four dwarfs and three control Friesian horses by Next Generation Sequencing. The DNA sequence of the critical region of ECA14 of the dwarfs was compared with those of the controls, the reference genome, and the Quarter Horse that is publicly available [6]. As dwarfism has not been reported in the Quarter Horse breed we assumed that the causative mutation is not present in this population and that the horse was homozygous for the reference allele. The variations of the dwarfs as compared with the reference genome were filtered by snpSift [7] for possible effects on amino acid sequence or splicing (Additional file 1). Using the Integrative Genomics Viewer [8] we then searched for the variations that were absent in the Quarter Horse and not homozygously present in the control Friesian horses. Only one nonsynonymous mutation fulfilled these criteria. The mutation was ECA14:g.4535550C $>\mathrm{T}$ in B4GALT7 and corresponds to XM_014730464.1:c.50G > A and XP_014585950.1:p.(Arg17Lys). Arginine and lysine are both basic amino acids that are interchangeably seen in many conserved protein domains. In this case, however, the arginine residue at position 17 of the equine B4GALT7 encoded protein xylosylprotein 


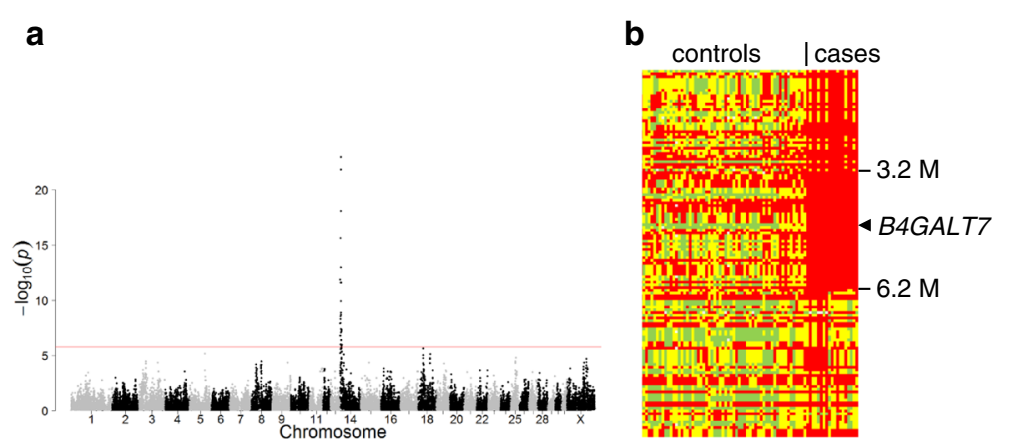

Fig. 2 Localization of the dwarfism locus in Friesian horses. a Manhattan plot of the genome wide association study. The DNA of 19 dwarfs and 65 controls of the Friesian horse breed was typed with the Illumina EquineSNP50 array. The allele frequency differences between the groups were assessed with GenABEL software and plotted as the - $\log _{10} p$ value from Chi-square tests. The red line indicates the Bonferroni corrected significance level. $\mathbf{b}$ Homozygosity mapping. Individual genotypes of informative SNPs from the telomeric region (0 - $10 \mathrm{Mb})$ of ECA14 of cases and controls were extracted from the array data. The results of the telomeric 134 SNPs of the p-arm that passed quality control are shown. Red: homozygous genotype of major allele in the dwarfs; green: homozygous genotype of minor allele; yellow: heterozygous genotype. The region of homozygosity in the dwarfs is bordered by the SNPs BIEC2-239119 and BIEC2-240544

beta 1,4-galactosyltransferase, polypeptide 7 (galactosyltransferase I) is strictly conserved in all vertebrates analyzed (Fig. 3a). Nonetheless, the mutation was considered moderate by the snpSift analysis and benign by PolyPhen-2 [9].

The association of the mutation with the dwarfism phenotype was evaluated by Sanger DNA sequencing. All 29 dwarfs of which DNA was available were homozygous for the mutation (Fig. 4c, line 2). The 8 obligate carriers were heterozygous (line 3) and of a group of 177
Friesian horses 22 were carrier of the mutation and 155 were homozygous for the reference allele (line 1).

\section{RNA analysis}

The mutated guanosine nucleotide is the last residue of exon 1 of B4GALT7 and the position of this first splice donor relative to the start codon of the gene is conserved in vertebrates (Fig. 3b). The nucleotide is second in the triplet coding for arginine and since this amino acid is conserved, the guanosine is conserved with it.

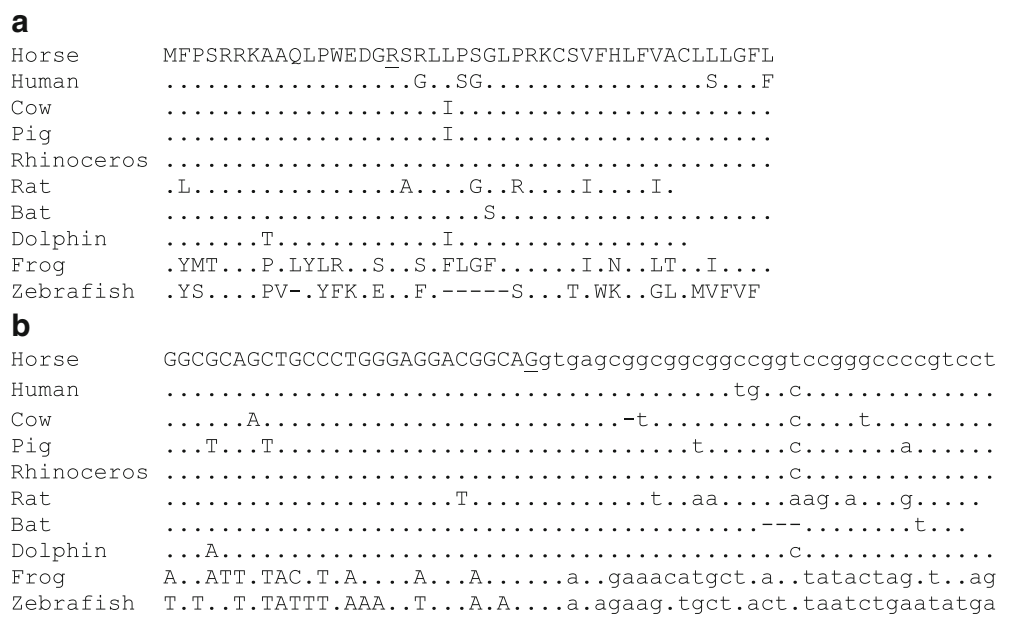

Fig. 3 Conservation of B4GALT7. a Alignment of the N-terminal amino acid sequence of xylosylprotein beta 1,4-galactosyltransferase, polypeptide 7 of horse with that of several mammals, an amphibian and a fish. The protein sequences are derived from the reference cDNAs listed below. $\mathbf{b}$ Alignment of the splice donor site of exon 1 of B4GALT7 of horse with that of other vertebrate species. The exon DNA sequence is in upper case and that of the intron is in lower case. The residues in $\mathbf{a}$ and $\mathbf{b}$ that are mutated in Friesian horse dwarfs are underlined. Residues that are identical to those in horse are indicated by a dot; gaps that are introduced to optimize the alignment are indicated by a dash. The exon 1/intron 1 splice junctions are derived from the respective reference genomes and based on the following CDNA reference sequences. Horse (Equus caballus): XM_014730464; human: NM_007255; cow (Bos taurus): NM_001075321; pig (Sus scrofa): NM_001 168422; rhinoceros (Ceratotherium simum): XM_010145472; rat (Rattus norvegicus): NM_001031661; bat (Eptesicus fuscus): XM_008143179; Dolphin (Tursiops truncatus): XM_004313659; frog [Xenopus (Silurana) tropicalis]: (NM_001 126545); zebrafish (Danio rerio): NM_001003417 


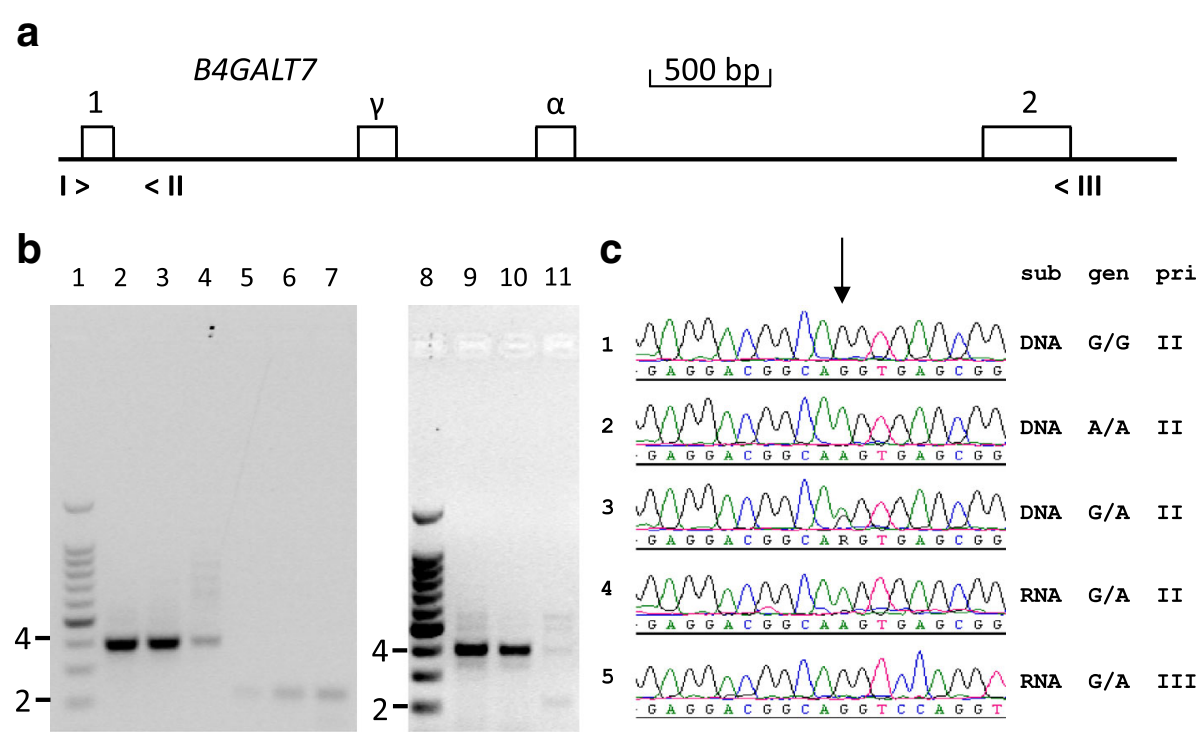

Fig. 4 Mutation of the terminal nucleotide of exon 1 of B4GALT7 affects proper RNA splicing of intron 1. a Map of the exon 1- exon 2 region of the equine B4GALT7 gene. The positions of the exons 1 and 2 are indicated by numbered boxes; $a$ : alternative exon; $\gamma$ : cryptic exon. The position of the used PCR primers are indicated by $>$ and < signs with Roman numerals. I>: EX1F; <II: IN1R; <III: EX2R. $\mathbf{b}$ CDNA fragments derived from a Friesian horse homozygous for the reference allele (lanes 2, 5 and 9), a heterozygous carrier (lanes 3.6 and 10) and a Friesian horse dwarf (lanes 4,7 and 11). Fibroblasts were grown from skin biopsies from the horses and RNA was isolated. cDNA was synthesized with reverse transcriptase followed by PCR with equine B4GALT7 specific primers. Lanes 1 and 8: 100 bp size standard ladder ( $2=200$ bp fragment, $4=400$ bp fragment); lanes 2-4: primers I and II; lanes 5-7: primers I and III; lanes 9-11: primers I, II and III. c Genomic DNA and RNA sequence analysis of B4GALT7 fragments from a horse homozygous for the reference allele $(\mathrm{G} / \mathrm{G})$, a dwarf (A/A) and a heterozygous carrier (G/A). The arrow indicates the position of the mutation. Sub: substrate genomic DNA (DNA) from the horses representing the three genotypes (gen) or cDNA synthesized with RNA from fibroblasts of the heterozygous horse (RNA). Pri: The PCR fragments were generated with primer I and either primer II or III as indicated. The mutant allele A is only observed in the unspliced RNA fragment (line 4) while the properly spliced product from the same heterozygous horse only shows the reference allele $\mathrm{G}$ (line 5)

We wondered whether the selection pressure could have worked the other way around; that is, that the nucleotide itself was conserved not due to its amino acid coding properties but due to its splicing function. The mutation of guanosine to adenosine might affect splicing of the primary RNA transcript of the gene and splicing requirements may block its propagation. According to the splice site predictor NNSPLICE 0.9 [10] the exon 1/intron 1 junction of the equine reference gene had a splice donor score of 0.96 on a scale of 0 to 1 . The mutated nucleotide sequence of the dwarfs had a moderate score of 0.58 , suggesting that the mutation could indeed interfere with splicing.

To investigate the effect of the mutation on splicing of the primary transcript we isolated RNA from cultured skin fibroblasts of a Friesian horse dwarf, of a heterozygous carrier of the B4GALT7 mutation and of a Friesian horse that was homozygous for the reference allele. Synthesis of cDNA was followed by PCR with a forward primer derived from B4GALT7 exon 1 and a reverse primer from intron 1 or exon 2 (Fig. 4a). The RNA of the wildtype horse and the heterozygous horse yielded a splicing product with the exonic primers of the expected length of $401 \mathrm{bp}$ (Fig. 4b, lanes 2 and 3). This product was less pronounced in the cDNA from the dwarf (lane 4). The primer set that included the intron 1 reverse primer produced a cDNA band derived from unspliced RNA of approximately $220 \mathrm{bp}$. This band was detected with the samples from the heterozygous horse and from the dwarf (lanes 6 and 7), but also, be it weakly, with the sample from the wildtype horse (lane 5). Combination of the 3 primers in a semi quantitative PCR showed spliced and unspliced products with similar intensities in the cDNA from the dwarf (lane 11). The wild type horse and the heterozygous horse do not display the unspliced product of $220 \mathrm{bp}$ with the 3 primer PCR (lanes 9 and 10).

When we analyzed the cDNA sequence of the unspliced product from the heterozygous horse (lane 6) it was derived from the mutant allele only (Fig. 4c, line 4). The cDNA sequence of the properly spliced product from the same horse (lane 3 ) indicated that it was derived from the normal allele (Fig. 4c, line 5). The B4GALT7 cDNA analysis confirms that the mutation r.50 g > a leads to a splicing deficiency of the primary transcript.

The exon 1/exon 2 primer set produced minor fragments that were larger than the expected length (Fig. 4b, lane 4). DNA sequence analysis of the fragments derived from the dwarf showed that the fragment of approximately 
560 bp contained an alternative exon of 162 nucleotides between exon 1 and exon 2 sequences that were spliced at the proper positions. This alternative exon is annotated as such in Genbank. It is situated from position 4533657 to 4533818 of chromosome 14 of the reference genome EquCab 2.0. It should be noted that the gene is situated in the reverse orientation on the chromosome. The alternative exon has an open reading frame that is in frame with the remainder of the coding sequence of the gene and the amino acid sequence encoded by the exon is conserved in mammals.

Another minor cDNA fragment of approximately 730 bp contained the same alternative exon sequence together with a cryptic exon of 169 nucleotides located between exon 1 and the alternative exon. The genomic location of the cryptic exon was from 4534375 to 4534543 on chromosome 14 and it contained multiple stopcodons in frame with the start codon on exon 1.

The aberrant splicing from the exon 1 donor due to the variant $\mathrm{r} .50 \mathrm{~g}>\mathrm{a}$ was associated with a severe reduction of expression of B4GALT7 at the mRNA level. Quantitative PCR measurement of cDNA fragments indicated that the concentration of transcripts from the gene in the fibroblasts from the dwarf was only $2 \%$ of that in fibroblasts from a Friesian horse that did not carry the mutation (Additional file 2).

\section{Discussion}

Disproportionate dwarfism in Friesian horses is associated with a mutation in B4GALT7. The mutation changes a conserved arginine codon to a lysine codon. Both amino acid residues are basic and the effect of the mutation is considered moderate by the snpSift prediction. The mutation also affects the last nucleotide of exon 1 of the gene. Unspliced cDNA fragments spanning the exon $1 /$ intron 1 junction can be detected regardless of the genotype of the horses. However, the cDNA sequences from a heterozygous horse clearly show that RNA derived from the mutant allele is hardly spliced, in contrast to the RNA from the normal allele (Fig. 4c, lines 4 and 5).

When an exonic and an intronic reverse primer are allowed to compete in a 3 primer PCR, only the cDNA from the dwarf displays the spliced and unspliced products in comparable amounts (Fig. 4b, lane 11). The normally spliced product is seen prominently in the wild type horse and the horse heterozygous for the mutation, but the unspliced product cannot be discerned among the products from these horses (lanes 9 and 10). This semi quantitative PCR and the cDNA sequence analysis of the products of the heterozygous horse confirms that the B4GALT7 mutation strongly reduces the splicing capacity of the exon $1 /$ intron 1 junction.
In the homozygous state, the mutation leads to low mRNA levels and the expression of the gene is strongly reduced as measured by qPCR. The improperly spliced transcripts could be prone to nonsense mediated decay.

The nucleotide sequence AGgt of the exon 1 splice junction of B4GALT7 and its position with regard to the start codon are highly conserved (Fig. 3b). One could argue that the last nucleotide of the exon is expected to be conserved if the encoded arginine residue would be essential for the function of the protein. This $G$ residue is the second nucleotide of the codon and all six triplets that code for arginine have a $G$ residue at the second position. Thus, if the arginine is conserved, the guanosine is conserved with it. The first position of the codon under consideration is a conserved A residue, while 4 of the 6 possible arginine codons start with a C. A functional restriction on the encoded arginine residue would therefore not necessarily lead to conservation of the A residue of the AGgt splice junction. The hypothetical mutation of the A residue to a $\mathrm{C}$ would only lead to a moderate drop of the splice donor score from 0.96 to 0.89 . According to this prediction a mutation of the second to last $A$ residue to a $C$ would be allowed while in fact it is highly conserved. Recently, a mutation of the A residue of a splice donor site in $I B A 57$ with the same AGgt junction sequence as exon 1 of B4GALT7 was shown to impede proper splicing, causing a severe leukoencephalopathy [11]. This mutation did not alter the encoded amino acid and it stresses the importance of the exonic terminal nucleotide sequences for splicing at particular junctions. An in vitro splicing assay may resolve the importance of the second to last A residue of the exon 1 of B4GALT7 for proper splicing. Concurrent with our results, the NNSPLICE program assigns a much lower splice donor score of 0.58 for the mutation found in the Friesian dwarfs. Considering all our results, we conclude that the conservation of the exon 1 terminal sequence in vertebrates reflects a restriction on a splicing requirement rather than on a functional requirement of the encoded amino acid. Characterization of naturally occurring mutations that are uncovered because of an association with disease can render important insights in splicing requirements [12].

In humans, mutations in B4GALT7 cause the EhlersDanlos syndrome, progeroid type 1 (EDSP1, OMIM130070) and Larsen of Reunion Island syndrome (LRS). Only $7 \mathrm{mu}-$ tations have been described in relation to the recessively inherited syndromes [13-18]. Most patients were normal with respect to length and weight at birth but soon were presented with growth retardation, osteopenia, facial dysmorphology, loose joints, bone dysplasias, loose skin and in most cases mild forms of mental retardation. Pectus carinatum was reported for a number of patients [16, 17]. The human phenotype is highly 
variable, even in patients sharing the same mutation homozygously [17].

A founder effect in a closed population of Reunion has led to at least 22 cases of LRS that were genetically confirmed. LRS was described as a subtype of Larsen syndrome [19]. The same mutation that causes LRS was observed homozygously in two siblings from another population diagnosed with EDSP1. The progeroid aspect was not observed in any of the genetically confirmed cases of EDSP1 nor LRS and it has been suggested to remove this term from the name of the EDSP1 syndrome [16, 18].

Clear similarities between the conditions in man and horse are growth retardation and hypermobile joints. Rib deformities have been observed in human as well as equine cases $[1,16,17]$. Pectus carinatum, reported in human cases, refers to the pectus in which the ribcartilage has been overdeveloped outward, leading to a 'chicken chest'. In the Friesian horse cases on the other hand, the ribcartilage has been overdeveloped inward, leading to pectus excavatum or 'shoemaker chest' in humans.

The dwarfism in the horse is described as a disproportionate growth disturbance because all limbs are short, while the head and back appear rather normal. In contrast, almost all confirmed human patients with LRS and EDSP1 display facial dysmorphism and disproportional growth was not noted [16, 17]. Cognitive functions do not seem to be impaired in dwarf horses. Another clear difference between the phenotype in man and horse is that loose skin has never been observed in Friesian dwarfs. Atrophic scarring and/or delayed wound healing has been reported for a number of human patients but is never seen in Friesian horse dwarfs. The fibroblasts from one human patient displayed reduced proliferation rates [20], while the fibroblasts of the Friesian dwarf proliferated at least as fast as the fibroblasts from normal Friesians. The differences in the clinical presentations between human patients and Friesian dwarfs may be due to the nature of the mutation in horses, which we think has predominantly an effect on the expression level of a normally functioning protein. On the other hand, the protein may have rate limiting key roles in processes that are different in the two species, such that loss of activity becomes manifest in different ways.

The B4GALT7 gene is highly expressed in the proliferative zone of the growth plate in rat [16]. Deficiency of the encoded xylosylprotein beta-1,4-galactosyltransferase 7 apparently induces the irregularities of the chondrocyte columns seen in the growth plate of Friesian dwarfs [1]. The enzyme adds the second of four saccharides that form the linker between the protein core and the glycosaminoglycan moiety of proteoglycans. Proteoglycans are major components of molecular networks of the extracellular matrix. Mutations in any of the enzymes that build the saccharide linker cause a variety of rare syndromes with overlapping features, which are called linkeropathies (reviewed in [21]). Dwarfism in Friesian horses could therefore be considered as a new presentation of a linkeropathy.

Remarkably, this is the second gene with a role in protein glycosylation in which we found a pathogenic mutation in Friesian horses. Earlier we found a nonsense mutation in B3GALNT2 involved in muscular dystrophy with hydrocephalus in stillborn foals [22]. The encoded beta-1,3-N-acetylgalactosaminyltransferase is involved in glycosylation of alpha-dystroglycan, which is part of the complex that connects the cytoskeleton with the extracellular matrix.

\section{Conclusions}

We provide evidence indicating that dwarfism in Friesian horses could be caused by a splicing deficiency of B4GALT7 that severely reduces expression of the gene. The conservation of the affected nucleotide reflects a splicing requirement rather than a functional requirement of the encoded amino acid. The clinical picture of the Friesian horse dwarfs adds to the phenotypic variability observed in human patients with B4GALT7 mutations. Crosses between carriers can be prevented by screening breeding horses for the B4GALT7 mutation and the dwarfism trait could thus be eliminated from the breed.

\section{Methods}

Phenotypes, genotypes and genome-wide association study Friesian horses $(n=29)$ were diagnosed as being dwarfs by local equine veterinarians in consultation with the Equine Clinic of Utrecht University, usually via a digital in vivo picture for confirmation of the phenotype. Thirteen of the horses were male, 11 female and the sex of 5 dwarfs was unknown. The group of unaffected controls $(n=65)$ were Friesian horses without the characteristic appearance of dwarfism [1]. In addition, we obtained blood samples for DNA isolation from 8 parents of dwarfs and DNA samples from 177 Friesian horses that were part of a DNA bank maintained at the Dr. Van Haeringen Laboratory B.V..

Blood samples were taken and DNA was isolated as described by Orr et al. [4]. Genotypes of 19 dwarfs and 65 controls were obtained using the Illumina ${ }^{\circ}$ EquineSNP50 Genotyping BeadChip containing 54,602 SNPs. Quality control was performed using the check.marker function in the GenABEL package in $\mathrm{R}$ [23]. SNPs with MAF $<5 \%$ and call-rate $<90 \%$ were discarded, leaving 29,840 SNPs (54.7\% of all SNPs) for the analysis. 
The ccfast function in GenABEL package in $\mathrm{R}$ [23] was used to determine the significance of allelic differences between dwarfs and unaffected horses with a $x^{2}$-test (1df). The Bonferroni corrected significance level applied was $1.68 \times 10^{-6}$. Homozygosity mapping in the significantly associated region was performed by eye to identify overlapping regions of homozygosity between dwarfs.

\section{Genome sequencing}

Four dwarf cases and three unrelated control were paired-end sequenced with 150 nucleotide reads for the full genome on Illumina NextSeq500 to an average coverage of 4-9x according to the manufacturers protocols. To increase the power to detect causal candidate variants as a fully homozygous variant, we merged the data for the four cases yielding an mean coverage of 36x.

The Illumina data was processed with our inhouse developed pipeline v 1.2.1 [24] including GATK v3.2.2 [25] according to the best practices guidelines [26]. Briefly, we mapped the pairs with BWA-MEM v0.7.5a [27], marked duplicates, merged lanes, realigned indels. Base recalibration did not improve our results, so this step was skipped. Next, GATK Haplotypecaller was used to call SNPs and indels. Variants are flagged as PASS only if they do not meet the following criteria: $\mathrm{QD}<2.0, \mathrm{MQ}<$ 40.0,FS > 60.0, HaplotypeScore $>13.0$, MQRankSum $<-12.5$, ReadPosRankSum $<-8.0$, snpclusters $>=3$ in 35 bp. For indels: $\mathrm{QD}<2.0, \mathrm{FS}>200.0$, ReadPosRankSum $<-20.0$.

Detecting recessive candidate variants was done with snpSift [28] fitting the model of reference or potential carrier status in the control and homozygous state in the cases. Moreover, coverage $>10$, a genotypequality of $>30$ and effect impact 'HIGH' or 'MODERATE' was required. Additional evaluation of the variant of interest was performed with PolyPhen-2 [9].

The observed possibly detrimental DNA variant of B4GALT7 was confirmed and evaluated in the complete cohort by Sanger sequencing of PCR fragments. The PCR primer sequences were 5'- AGTTTCTCGGAG TGTAGAG-3' (UP1F) and 5'-AGAGACATAGACCCTC AGAG-3' (IN1R). The PCR was performed with 50 ng genomic DNA, $3 \mathrm{U}$ Platinum Taq DNA polymerase (Thermo Fisher Scientific, Waltham, MA), $2 \mathrm{mM}$ $\mathrm{MgCl} 2,0.2 \mathrm{mM}$ each dNTP, $0.5 \mu \mathrm{M}$ each primer, $1 \mathrm{M}$ betaine and $1 \times$ Platinum buffer. Temperature cycling conditions were $5 \mathrm{~min}$ at $95^{\circ} \mathrm{C}, 35$ cycles of $30 \mathrm{~s}$ at $95^{\circ}$ $\mathrm{C}, 30 \mathrm{~s}$ at $55^{\circ} \mathrm{C}, 30 \mathrm{~s}$ at $72{ }^{\circ} \mathrm{C}$, and a final elongation step at $72{ }^{\circ} \mathrm{C}$ for $10 \mathrm{~min}$. All amplifications were performed on an ABI 9700 Thermal Cycler (Applied Biosystems, Foster City, CA). The PRC primers were degraded by addition of $1 \mathrm{U}$ Exonuclease I (Thermo Fisher Scientific, Waltham, MA) and incubation for $15 \mathrm{~min}$. at $37^{\circ} \mathrm{C}$ and $15 \mathrm{~min}$. at $85{ }^{\circ} \mathrm{C}$. DNA sequencing tercycle reactions were performed using BigDye v3.1 (Thermo Fisher Scientific, Waltham, MA) according to the manufacturer's protocol. The products were analysed on a 3130XL Genetic Analyzer (Applied Biosystems, Foster City, CA) and the data was analysed with Lasergene (version 11 DNASTAR).

Homologous DNA and protein sequences from different species were retrieved from GenBank and aligned one by one by eye. Identities and differences were indicated by using a word processor. The species were selected arbitrary to represent close and distant members of the animal kingdom.

\section{RNA analysis}

Fibroblasts were grown from $6 \mathrm{~mm}$ punch biobsies from the skin of a dwarf, a carrier of the mutation of interest and a Friesian horse homozygous for the reference allele. The biopsies were washed in Euroflush (IMV technologies, L'Aigle, France) containing $5000 \mathrm{IU} / \mathrm{ml}$ heparin, cut with scissors and incubated in petri dishes with DMEM/ M199 (1:1) medium containing pen/strep (10,000 U/ml (all from Thermo Fisher Scientific, Waltham, MA), $2.5 \mathrm{ng} / \mathrm{ml}$ basic-FGF5 (Peprotech, Rocky Hill, NJ) and $20 \%$ FCS at $38.5{ }^{\circ} \mathrm{C}$ with $5 \% \mathrm{CO}_{2}$ and $5 \% \mathrm{O}_{2}$. Proliferating fibroblasts were harvested and passaged in culture flasks using standard procedures. RNA was isolated from cultured fibroblasts with the RNeasy kit with an oncolumn DNase digestion according to the instructions of the manufacturer (Qiagen, Hilden, Germany). The RIN value of the RNA was meausured with an Agilent 2100 Bioanalyzer (Santa Clara, CA, USA) and was found to be 9.5 or higher. cDNA was synthesized with the iScript kit (Bio-Rad Laboratories, Hercules, CA) using $500 \mathrm{ng}$ of RNA in $20 \mu \mathrm{l}$ reactions. Splicing products were PCR amplified from $0.5 \mu \mathrm{l}$ cDNA product with B4GALT7 exonic primers 5'-CTGGGAGCTCGAGCTCCATG-3' (EX1F) and 5'-CTCAGGAAGCGGTGCATGTG-3' (EX2R) as described above. Unspliced products were amplified with primer EX1F and the intronic primer IN1R described above. In a semi quantitative experiment, the 3 primers were combined in a single PCR using the same component concentrations and cycling conditions as above. The fragments were visualized by electrophoresis on a $1 \%$ agarose gel in $0.5 \mathrm{x}$ TBE with $0.5 \mu \mathrm{g} / \mathrm{ml}$ ethidium bromide followed by UV irradiation of the gel.

For DNA sequence analysis and confirmation of the origin of the products, the bands were cut from the gel and the DNA was isolated with QIAquick gel extraction kit (Qiagen, Hilden, Germany). The DNA sequencing procedure was as described above.

Quantitative PCR for measurement of B4GALT7 transcripts was performed with forward primer 5'- G ACGGCAGGTCCAGGTTG-3' and reverse primer 5'- ACAGGCAACGAAGAGGTGG-3' at an annealing 
temperature of $55^{\circ} \mathrm{C}$. The forward primer bridges exons 1 and 2, while the reverse primer is situated in exon 2. The qPCR reactions contained 1x IQ SYBRGreen SuperMix (Bio-Rad, Laboratories, Hercules, CA), $400 \mathrm{nM}$ of each primer and $1 \mu \mathrm{l} \mathrm{cDNA}$, obtained as described above, in a total volume of $15 \mu \mathrm{l}$. The reference genes were RPS19 and RPL13A, which were analyzed as described [29]. The reactions were performed in a MyiQ2 thermal cycler and the data was analyzed with IQ5 software (both from BioRad, Laboratories, Hercules, CA).

\section{Additional files}

Additional file 1: Variants with predicted moderate or high effect on gene function in the critical chromosome region of Friesian dwarf horses. Output of snpSift analysis of Next Generation Sequence data. (XLSX 15 kb)

Additional file 2: Expression of B4GALT7 in relation to genotype. qPCR data of B4GALT7 mRNA from fibroblasts. (XLSX $13 \mathrm{~kb}$ )

\section{Abbreviations}

ECA14: Equus Caballus chromosome 14; EDSP1: Ehlers-Danlos syndrome, progeroid type 1; KFPS: Koninklijke Vereniging "Het Friesch Paarden-Stamboek" (i.e. Royal Friesian Horse Studbook); LRS: Larsen of Reunion Island syndrome.

\section{Acknowledgements}

The photos of Fig. 1 were provided by the Multimedia Department, Faculty of Veterinary Medicine, Utrecht University. We gratefully acknowledge the assistence of Mrs. Yteke Elte of the Department of Equine Sciences of Utrecht University with taking the skin biopsies. We are very grateful to the owners of the horses that were part of this study.

\section{Funding}

This study was funded in part by the KFPS. This organization also identified Friesian horses of interest to the study and facilitated the collection of samples.

\section{Availability of data and materials}

The whole genome sequence data is available at the European Nucleotide Archive (ENA) [30] under study accession number PRJEB13863.

\section{Authors' contributions}

PAL, BJD, IH, WB and AS conceived and designed the experiments. MV- $L, I J B$, FGVS, GRM, JWMB, BWD and LHVdG performed the experiments. PAL, BJD, IJN and AS analyzed the data. $I H$ and WB contributed materials. PAL and AS wrote the manuscript. All authors read and approved the manuscript.

\section{Competing interests}

LHvdG is employed by Dr. Van Haeringen Laboratorium B.V. that performs DNA diagnostic tests on a commercial basis. $I H$ is director of the studbook office of the KFPS (Koninklijke Vereniging "Het Friesch Paarden-Stamboek" i.e. Royal Friesian Horse Studbook).

\section{Consent for publication}

Not applicable.

\section{Ethics approval}

The horses that were included in the genome wide mapping, Next Generation Sequencing and validation experiments were privately owned and blood samples for DNA isolation were taken with informed consent from the owners. The skin biopsies were taken from University Utrecht owned Friesian teaching horses. Blood and skin sampling was done with approval of the Animal Ethics Committee of the Utrecht University (DEC file 2013.|II.01.012)

\section{Author details}

'Department of Clinical Sciences of Companion Animals, Faculty of Veterinary Medicine, Utrecht University, PO Box 80154NL-3508 TD Utrecht, The Netherlands. ${ }^{2}$ Animal Breeding and Genomics Centre, Wageningen University, PO Box 338NL-6700 AH Wageningen, The Netherlands. ${ }^{3}$ Department of Equine Sciences, Faculty of Veterinary Medicine, Utrecht University, Yalelaan 112-114, NL-3584 CM Utrecht, The Netherlands. ${ }^{4}$ Department of Medical Genetics, University Medical Center Utrecht, PO Box $85090 N L-3508$ AB Utrecht, The Netherlands. ${ }^{5}$ Dr. van Haeringen Laboratorium B.V., PO Box 408NL-6700 AK Wageningen, The Netherlands. ${ }^{6}$ Koninklijke Vereniging "het Friesch Paarden-Stamboek", PO Box 624NL-9200 AP Drachten, The Netherlands. ${ }^{7}$ Department of Surgery and Anaesthesiology of Domestic Animals, Faculty of Veterinary Medicine, Ghent University, Salisburylaan 133, B-9820 Merelbeke, Belgium.

Received: 14 May 2016 Accepted: 22 October 2016

Published online: 28 October 2016

\section{References}

1. Back W, Van der Lugt JJ, Nikkels PGJ, Van den Belt AJM, Van der Kolk JH, Stout TAE. Phenotypic diagnosis of dwarfism in six Friesian horses. Equine Vet J. 2008:40:282-7.

2. University of Sydney: OMIA - Online Mendelian Inheritance in Animals. http://omia.angis.org.au/home/. Accessed 7 Dec 2015.

3. De Graaf-Roelfsema E, Back W, Keizer HA, Stout TA, van der Kolk JH. Normal function of the hypothalamic-pituitary growth axis in three dwarf Friesian foals. Vet Rec. 2009;165:373-6.

4. Orr N, Back W, Gu J, Leegwater P, Govindarajan P, Conroy J, Ducro B, Van Arendonk JAM, MacHugh DE, Ennis S, Hill EW, Brama PA. Genome-wide SNP association-based localization of a dwarfism gene in Friesian dwarf horses. Anim Genet. 2010;41 Suppl 2:2-7.

5. NCBI: map viewer. http://www.ncbi.nlm.nih.gov/projects/mapview/map_ search.cgi?taxid=9796\&build=101.0. Accessed 7 Dec 2015.

6. Doan R, Cohen ND, Sawyer J, Ghaffari N, Johnson CD, Dindot SV. Wholegenome sequencing and genetic variant analysis of a Quarter Horse mare. BMC Genomics. 2012;13:78.

7. Kumar P, Henikoff $\mathrm{S}, \mathrm{Ng}$ PC. Predicting the effects of coding nonsynonymous variants on protein function using the SIFT algorithm. Nat Protoc. 2009:4:1073-81.

8. Robinson JT, Thorvaldsdóttir H, Winckler W, Guttman M, Lander ES, Getz G, Mesirov JP. Integrative Genomics Viewer. Nat Biotechnol. 2011;29:24-6.

9. Adzhubei IA, Schmidt S, Peshkin L, Ramensky VE, Gerasimova A, Bork P, Kondrashov AS, Sunyaev SR. A method and server for predicting damaging missense mutations. Nat Methods. 2010:7:248-9.

10. Reese MG, Eeckman FH, Kulp D, Haussler D. Improved Splice Site Detection in Genie. J Comp Biol. 1997:4:311-23.

11. Lossos A, Stümpfig C, Stevanin G, Gaussen M, Zimmerman BE, Mundwiller E, Asulin M, Chamma L, Sheffer R, Misk A, Dotan S, Gomori JM, Ponger P, Brice A, Lerer I, Meiner $V$, Lill R. Fe/S protein assembly gene IBA57 mutation causes hereditary spastic paraplegia. Neurology. 2015;84:659-67.

12. Voorbij AM, Van Steenbeek FG, Vos-Loohuis M, Martens EE, Hanson-Nilsson JM, Van Oost BA, Kooistra HS, Leegwater PA. A contracted DNA repeat in LHX3 intron 5 is associated with aberrant splicing and pituitary dwarfism in German shepherd dogs. PLoS One. 2011;6:e27940.

13. Almeida R, Levery SB, Mandel U, Kresse H, Schwientek T, Bennett EP, Clausen $\mathrm{H}$. Cloning and expression of a proteoglycan UDP-galactose:betaxylose beta-1,4-galactosyltransferase I: a seventh member of the human beta4-galactosyltransferase gene family. J Biol Chem. 1999;274:26165-71.

14. Okajima T, Fukumoto S, Furukawa K, Urano T, Furukawa K. Molecular basis for the progeroid variant of Ehlers-Danlos syndrome: identification and characterization of two mutations in galactosyltransferase I gene. J Biol Chem. 1999;274:28841-4

15. Faiyaz-Ul-Haque M, Zaidi SHE, Al-Ali M, Al-Mureikhi MS, Kennedy S, Al-Thani G, Tsui L-C, Teebi AS. A novel missense mutation in the galactosyltransferase-I (B4GALT7) gene in a family exhibiting facioskeletal anomalies and Ehlers-Danlos syndrome resembling the progeroid type. Am J Med Genet A. 2004;128A:39-45

16. Guo MH, Stoler J, Lui J, Nilsson O, Bianchi DW, Hirschhorn JN, Dauber A Redefining the progeroid form of Ehlers-Danlos syndrome: report of the fourth patient with B4GALT7 deficiency and review of the literature. Am J Med Genet A. 2013;161A:2519-27. 
17. Cartault F, Munier P, Jacquemont ML, Vellayoudom J, Doray B, Payet C, Randrianaivo H, Laville JM, Munnich A, Cormier-Daire V. Expanding the clinical spectrum of B4GALT7 deficiency: homozygous p.R270C mutation with founder effect causes Larsen of Reunion Island syndrome. Eur I Hum Genet. 2015;23:49-53.

18. Salter CG, Davies JH, Moon RJ, Fairhurst J, Bunyan D; DDD Study, Foulds N. Further defining the phenotypic spectrum of B4GALT7 mutations. Am J Med Genet A. 2016;doi:10.1002/ajmg.a.37604.

19. Laville JM, Lakermance P, Limouzy F. Larsen's syndrome: review of the literature and analysis of thirty-eight cases. J Pediatr Orthop. 1994;14:63-73.

20. Seidler DG, Faiyaz-UI-Haque M, Hansen U, Yip GW, Zaidi SH, Teebi AS, Kiesel L, Götte M. Defective glycosylation of decorin and biglycan, altered collagen structure, and abnormal phenotype of the skin fibroblasts of an EhlersDanlos syndrome patient carrying the novel Arg270Cys substitution in galactosyltransferase I (beta4GalT-7). J Mol Med (Berl). 2006;84:583-94.

21. Jones KL, Schwarze U, Adam MP, Byers PH, Mefford HC. A homozygous B3GAT3 mutation causes a severe syndrome with multiple fractures, expanding the phenotype of linkeropathy syndromes. Am J Med Genet A. 2015;167A:2691-6.

22. Ducro BJ, Schurink A, Bastiaansen JWM, Boegheim IJ, van Steenbeek FG, Vos-Loohuis M, Nijman IJ, Monroe GR, Hellinga I, Dibbits BW, Back W, Leegwater PA. A nonsense mutation in B3GALNT2 is concordant with hydrocephalus in Friesian horses. BMC Genomics. 2015;16:761.

23. Aulchenko YS, Ripke S, Isaacs A, van Duijn CM. GenABEL: an R library for genome-wide association analysis. Bioinformatics. 2007;23:1294-6.

24. University Medical Center Utrecht: Illumina analysis pipeline. https://github. com/CuppenResearch/IAP. Accessed 7 Dec 2015

25. McKenna A, Hanna M, Banks E, Sivachenko A, Cibulskis K, Kernytsky A, Garimella K, Altshuler D, Gabriel S, Daly M, DePristo MA. The Genome Analysis Toolkit: a MapReduce framework for analyzing next-generation DNA sequencing data. Genome Res. 2010;20:1297-303.

26. Van der Auwera GA, Carneiro M, Hartl C, Poplin R, del Angel G, LevyMoonshine A, Jordan T, Shakir K, Roazen D, Thibault J, Banks E, Garimella K, Altshuler D, Gabriel S, DePristo M. From FastQ Data to High-Confidence Variant Calls: The Genome Analysis Toolkit Best Practices Pipeline. Curr Protoc Bioinformatics. 2013:11:11.10.1-11.10.33.

27. Li H, Durbin R. Fast and accurate short read alignment with BurrowsWheeler Transform. Bioinformatics. 2009;25:1754-60.

28. Cingolani P, Platts A, le Wang L, Coon M, Nguyen T, Wang L, Land SJ, Lu X, Ruden DM. A program for annotating and predicting the effects of single nucleotide polymorphisms, SnpEff: SNPs in the genome of Drosophila melanogaster strain w1118; iso-2; iso-3. Fly (Austin). 2012;6:80-92.

29. Stassen QE, Riemers FM, Reijmerink H, Leegwater PA, Penning LC. Reference genes for reverse transcription quantitative PCR in canine brain tissue. BMC Res Notes. 2015;8:761.

30. EMBL-EBI: European Nucleotide Archive. http://www.ebi.ac.uk/ena. Accessed 4 May 2016.

\section{Submit your next manuscript to BioMed Central and we will help you at every step:}

- We accept pre-submission inquiries

- Our selector tool helps you to find the most relevant journal

- We provide round the clock customer support

- Convenient online submission

- Thorough peer review

- Inclusion in PubMed and all major indexing services

- Maximum visibility for your research

Submit your manuscript at www.biomedcentral.com/submit

) Biomed Central 